\title{
Positive solutions for integral boundary value problems of fractional differential equations with delay
}

\author{
Shuai Li ${ }^{1}$, Zhixin Zhang ${ }^{1 *}$ and Wei Jiang
}

"Correspondence:

zhang_zhi_x@sina.com

${ }^{1}$ School of Mathematical Sciences,

Anhui University, Hefei, P.R. China

\section{Springer}

\begin{abstract}
In this article, a class of integral boundary value problems of fractional delayed differential equations is discussed. Based on the Guo-Krasnoselskii theorem, some existence results on the positive solutions are derived. Two simple examples are given to show the validity of the conditions of our main theorems.
\end{abstract}

Keywords: Positive solutions; Fractional differential equations; Caputo derivative; Delay; Integral boundary value problems

\section{Introduction}

Differential equation models have been widely used in control system, aerodynamics, fluid flows and many other branches of engineering [1-9]. Recently, fractional calculus has attracted great interest. There are several kinds of fractional operators have been proposed so far, among which we have the well-known Grünwald-Letnikov, Riemann-Liouville, Caputo derivative.

In the past several decades, fractional boundary value problems have obtained abundant theoretical achievements. In $[10,11]$, the nonexistence of positive solutions for differential equations of fractional order is analyzed with the help of reduction to absurdity. In [12], the authors study a class of Riemann-Liouville fractional derivative equations and present sufficient condition on the unique positive solution by employing a $u_{0}$-positive operator. There are many articles devoted to the existence and multiplicity of positive solutions for the fractional boundary value problems, the approaches mainly include Leray-Schauder degree theory $[13,14]$, the monotone iterative method $[15,16]$, the Leggett-Williams theorem $[17,18]$, the fixed point theorem on cones [17-19]. Especially, compared with the previous results, papers [11-13, 17, 20] contain integral boundary conditions. In [21], the authors first introduced a new method, called Avery-Peterson theory, which illustrates the existence of at least three positive solutions. Since then, more and more attention [22-24] was paid to this method, and many questions were solved.

At present, fractional delayed equations have aroused the extensive attention of many scholars. They dealt with the existence of solutions under various boundary conditions by different methods. For details, one can refer to [20, 25-27] and the references therein.

(c) The Author(s) 2020. This article is licensed under a Creative Commons Attribution 4.0 International License, which permits use sharing, adaptation, distribution and reproduction in any medium or format, as long as you give appropriate credit to the original author(s) and the source, provide a link to the Creative Commons licence, and indicate if changes were made. The images or other third party material in this article are included in the article's Creative Commons licence, unless indicated otherwise in a credit line to the material. If material is not included in the article's Creative Commons licence and your intended use is not permitted by statutory regulation or exceeds the permitted use, you will need to obtain permission directly from the copyright holder. To view a copy of this licence, visit http://creativecommons.org/licenses/by/4.0/. 
In [28], Cabada and Wang considered a class of nonlinear fractional differential equations with integral boundary value conditions:

$$
\left\{\begin{array}{l}
{ }^{c} D^{\alpha} u(t)+f(t, u(t))=0, \quad 0<t<1, \\
u(0)=u^{\prime \prime}(0)=0, \quad u(1)=\lambda \int_{0}^{1} u(s) d s,
\end{array}\right.
$$

where $2<\alpha<3,0<\lambda<2, f:[0,1] \times[0, \infty) \rightarrow[0, \infty)$ is a continuous function. The existence of at least one positive solution is obtained by using the Guo-Krasnoselskii fixed point theorem.

Enlightened by the above literature, we discuss the following equation:

$$
\left\{\begin{array}{l}
{ }^{c} D^{\beta} z(t)+g\left(t, z_{t}\right)=0, \quad t \in[0,1], \\
z(t)=\phi(t), \quad t \in[-\tau, 0], \\
z(0)=z^{\prime \prime}(0)=z^{\prime \prime \prime}(0)=0, \quad z(1)=k \int_{0}^{1} z(\theta) d \theta,
\end{array}\right.
$$

where $3<\beta \leq 4,0<k<2,{ }^{c} D^{\beta}$ is the Caputo fractional derivative, $g:[0,1] \times C_{\tau} \longrightarrow$ $[0,+\infty)$ is a continuous function, $z_{t}(s)=z(t+s)$, for $t \in[0,1], s \in[-\tau, 0] . \phi \in C_{\tau}(:=$ $C[-\tau, 0]), C_{\tau}$ is a Banach space with $\|\phi\|_{[-\tau, 0]}=\max _{s \in[-\tau, 0]}|\phi(s)|$ and let $C_{\tau}^{+}=\{z \in$ $C[-\tau, 0] \mid z(t) \geq 0, t \in[-\tau, 0]\}$.

In this paper, the sufficient conditions are obtained for the existence of at least two positive solutions for a class of integral boundary value problems of fractional differential equations with delay. The integral boundary value condition and the time delay make the results significant.

\section{Preliminaries}

This part introduce some useful definitions and important lemmas.

Definition 2.1 ([1]) The $\beta$ order fractional integral for a function $g(t)$ is defined as follows:

$$
I^{\beta} g(t)=\frac{1}{\Gamma(\beta)} \int_{0}^{t}(t-\theta)^{\beta-1} g(\theta) d \theta, \quad \beta>0 .
$$

Definition 2.2 ([1]) The $\beta$ order Caputo fractional derivative for a function $g(t)$ is defined as follows:

$$
{ }^{c} D^{\beta} g(t)=\frac{1}{\Gamma(n-\beta)} \int_{0}^{t}(t-\theta)^{n-\beta-1} g^{(n)}(\theta) d \theta, \quad n-1<\beta \leq n .
$$

Definition 2.3 ([2]) Let $P \subseteq X$ be a nonempty, convex closed set and $X$ a real Banach space. The $P$ is called a cone in $X$ provided that

(i) $\mu z \in P$, for all $z \in P$ and $\mu \geq 0$;

(ii) $z,-z \in P$ imply $z=0$.

Definition 2.4 ([2]) Let $P$ is a cone in real Banach space $X$. If map $\psi: P \rightarrow[0, \infty)$ is continuous and satisfies

$$
\psi\left(t z_{1}+(1-t) z_{2}\right) \geq t \psi\left(z_{1}\right)+(1-t) \psi\left(z_{2}\right), \quad z_{1}, z_{2} \in P, t \in[0,1]
$$

then $\psi$ is called a nonnegative continuous concave functional on $P$. 
Lemma 2.1 ([1]) Assume that $\beta>0$ and $n=[\beta]+1$. If the function $g \in L[0,1] \cap C[0,1]$, then there exists $c_{i} \in R, i=1,2, \ldots, n$, such that

$$
I^{\beta c} D^{\beta} g(t)=g(t)-c_{1}-c_{2} t-\cdots-c_{n} t^{n-1} .
$$

Lemma 2.2 Equation(1) has a unique solution as follows:

$$
z(t)= \begin{cases}\int_{0}^{1} G(t, \theta) g\left(\theta, z_{\theta}\right) d \theta, & t \in[0,1] \\ \phi(t), & t \in[-\tau, 0]\end{cases}
$$

where

$$
G(t, \theta)= \begin{cases}\frac{2 t(1-\theta)^{\beta-1}(\beta-k+k \theta)-(2-k) \beta(t-\theta)^{\beta-1}}{(2-k) \Gamma(\beta+1)}, & 0 \leq \theta \leq t \leq 1, \\ \frac{2 t(1-\theta)^{\beta-1}(\beta-k+k \theta)}{(2-k) \Gamma(\beta+1)}, & 0 \leq t \leq \theta \leq 1 .\end{cases}
$$

Proof From Lemma 2.1, we have

$$
z(t)=-\frac{1}{\Gamma(\beta)} \int_{0}^{t}(t-\theta)^{\beta-1} g\left(\theta, z_{\theta}\right) d \theta+c_{0}+c_{1} t+c_{2} t^{2}+c_{3} t^{3} .
$$

According to $z(0)=z^{\prime \prime}(0)=z^{\prime \prime \prime}(0)=0, z(1)=k \int_{0}^{1} z(\theta) d \theta$, we know that

$$
c_{0}=c_{2}=c_{3}=0, \quad c_{1}=\frac{1}{\Gamma(\beta)} \int_{0}^{1}(1-\theta)^{\beta-1} g\left(\theta, z_{\theta}\right) d \theta+k \int_{0}^{1} z(\theta) d \theta .
$$

Thus, Eq. (1) has a unique solution

$$
\begin{aligned}
z(t)= & -\frac{1}{\Gamma(\beta)} \int_{0}^{t}(t-\theta)^{\beta-1} g\left(\theta, z_{\theta}\right) d \theta \\
& +\left(\frac{1}{\Gamma(\beta)} \int_{0}^{1}(1-\theta)^{\beta-1} g\left(\theta, z_{\theta}\right) d \theta+k \int_{0}^{1} z(\theta) d \theta\right) t .
\end{aligned}
$$

Letting $J=\int_{0}^{1} z(\theta) d \theta$, from (4) we get

$$
\begin{aligned}
J= & \int_{0}^{1} z(t) d t \\
= & -\int_{0}^{1} \int_{0}^{t} \frac{(t-\theta)^{\beta-1}}{\Gamma(\beta)} g\left(\theta, z_{\theta}\right) d \theta d t \\
& +\int_{0}^{1} \int_{0}^{1} \frac{t(1-\theta)^{\beta-1}}{\Gamma(\beta)} g\left(\theta, z_{\theta}\right) d \theta d t+\int_{0}^{1} k J t d t \\
= & -\int_{0}^{1} \frac{(1-\theta)^{\beta}}{\beta \Gamma(\beta)} g\left(\theta, z_{\theta}\right) d \theta+\frac{1}{2} \int_{0}^{1} \frac{(1-\theta)^{\beta-1}}{\Gamma(\beta)} g\left(\theta, z_{\theta}\right) d \theta+\frac{1}{2} k J .
\end{aligned}
$$

It follows that

$$
J=-\frac{2}{2-k} \int_{0}^{1} \frac{(1-\theta)^{\beta}}{\beta \Gamma(\beta)} g\left(\theta, z_{\theta}\right) d \theta+\frac{1}{2-k} \int_{0}^{1} \frac{(1-\theta)^{\beta-1}}{\Gamma(\beta)} g\left(\theta, z_{\theta}\right) d \theta .
$$


Substituting (5) into (4), we derive

$$
\begin{aligned}
z(t)= & -\int_{0}^{t} \frac{(t-\theta)^{\beta-1}}{\Gamma(\beta)} g\left(\theta, z_{\theta}\right) d \theta+\int_{0}^{1} \frac{t(1-\theta)^{\beta-1}}{\Gamma(\beta)} g\left(\theta, z_{\theta}\right) d \theta \\
& -\frac{2 k}{2-k} \int_{0}^{1} \frac{t(1-\theta)^{\beta}}{\beta \Gamma(\beta)} g\left(\theta, z_{\theta}\right) d \theta+\frac{k}{2-k} \int_{0}^{1} \frac{t(1-\theta)^{\beta-1}}{\Gamma(\beta)} g\left(\theta, z_{\theta}\right) d \theta \\
= & -\int_{0}^{t} \frac{(t-\theta)^{\beta-1}}{\Gamma(\beta)} g\left(\theta, z_{\theta}\right) d \theta+\int_{0}^{1} \frac{2 t(1-\theta)^{\beta-1}(\beta-k+k \theta)}{(2-k) \Gamma(\beta+1)} g\left(\theta, z_{\theta}\right) d \theta \\
= & \int_{0}^{1} G(t, \theta) g\left(\theta, z_{\theta}\right) d \theta .
\end{aligned}
$$

The conclusion have been proved.

Lemma 2.3 ([28]) The function $G(t, \theta)$ satisfies

(1) $0<G(t, \theta) \leq \frac{2}{(2-k) \Gamma(\beta)}$ for $t, \theta \in(0,1)$ if and only if $0<k<2$;

(2) $t G(1, \theta) \leq G(t, \theta) \leq M G(1, \theta), M=\frac{2 \beta}{k(\beta-2)}$ for all $t, \theta \in(0,1), 3<\beta \leq 4$ and $0<k<2$.

Lemma 2.4 ([2]) Suppose that $P$ is a cone in Banach space $X$. If $\Omega_{1}, \Omega_{2}$ are bounded open sets in $X$ such that $0 \in \Omega_{1}, \overline{\Omega_{1}} \subset \Omega_{2}$ and operator $T: P \cap\left(\overline{\Omega_{2}} \backslash \Omega_{1}\right) \rightarrow P$ is completely continuous satisfying

(i) $\|T z\| \geq\|z\|, z \in P \cap \partial \Omega_{1}$ and $\|T z\| \leq\|z\|, z \in P \cap \partial \Omega_{2}$; or

(ii) $\|T z\| \leq\|z\|, z \in P \cap \partial \Omega_{1}$ and $\|T z\| \geq\|z\|, z \in P \cap \partial \Omega_{2}$,

then the operator $T$ has at least one fixed point in $P \cap\left(\overline{\Omega_{2}} \backslash \Omega_{1}\right)$.

Lemma 2.5 ([29]) Suppose $\sigma \in\left(0, \frac{1}{2}\right)$ is a fixed number, for each $z \in P$ and $\theta \in[\sigma, 1-\sigma]$ ( $P$ is defined in Lemma 3.1), there exists a constant $\lambda \in(0,1)$ that satisfies

$$
\left\|z_{\theta}\right\|_{[-\tau, 0]} \geq \lambda\|z\|_{[0,1]}, \quad\|z\|_{[0,1]}=\sup _{t \in[0,1]}|z(t)|
$$

\section{Main results}

Next, the problem of positive solutions for Eq. (1) are studied. For convenience, some notations and hypotheses are presented as follows:

$$
\begin{aligned}
& g_{\infty}=\lim _{z \in C_{\tau}^{+},\|z\|_{[-\tau, 0]} \rightarrow+\infty} \frac{g(t, z)}{\|z\|_{[-\tau, 0]}}, \quad g_{0}=\lim _{z \in C_{\tau}^{+},\|z\|_{[-\tau, 0]} \rightarrow 0^{+}} \frac{g(t, z)}{\|z\|_{[-\tau, 0]}}, \\
& A=\frac{\lambda}{2} \int_{\sigma}^{1-\sigma} G(1, \theta) d \theta, \lambda \in(0,1), \sigma \in\left(0, \frac{1}{2}\right), \quad B=M \int_{0}^{1} G(1, \theta) d \theta ;
\end{aligned}
$$

$\left(C_{1}\right) \phi(t) \geq 0$ on $[-\tau, 0]$

$\left(C_{2}\right) g(t, z) \geq 0$ for $t \in[0,1]$ and $z \in C_{\tau}^{+}$;

$\left(C_{3}\right) g_{0}=g_{\infty}=+\infty$;

$\left(C_{4}\right) g_{0}=g_{\infty}=0$;

$\left(C_{5}\right)$ if there exists a constant $m \geq\|\phi\|_{[-\tau, 0]}>0$, then

$$
g(t, z) \leq \frac{m}{B}, \quad\|z\|_{[-\tau, 0]} \in[0, m], t \in[0,1] .
$$


$\left(C_{6}\right)$ if there exists a constant $n \geq\|\phi\|_{[-\tau, 0]}>0$, then

$$
g(t, z) \geq \frac{\lambda n}{A}, \quad\|z\|_{[-\tau, 0]} \in[\lambda n, n], t \in[\sigma, 1-\sigma] .
$$

On $C[-\tau, 1]$ define an operator $T$

$$
T z(t)= \begin{cases}\int_{0}^{1} G(t, \theta) g\left(\theta, z_{\theta}\right) d \theta, & t \in[0,1] \\ \phi(t), & t \in[-\tau, 0]\end{cases}
$$

Lemma 3.1 If $\left(C_{1}\right),\left(C_{2}\right)$ hold and $P$ is a cone in Banach space $X=C[-\tau, 1]$ with norm $\|z\|_{[-\tau, 1]}=\max _{t \in[-\tau, 1]}|z(t)|$ as follows:

$$
P=\{z \in X \mid z \geq 0, z \text { is concave down on }[0,1]\}
$$

then the following conclusions are true.

(i) $T(P) \subseteq P$;

(ii) $T: P \rightarrow P$ is completely continuous.

Proof It is easy to check that (i) holds and $T$ is continuous. So we only prove that (ii) is true. Assume that $H$ be a bounded subset in $P$, which is to say there exists $l>0$ such that $\|z\| \leq l$ for all $z \in H$. Let

$$
N=\sup _{t \in[0,1], z \in[0, l]}\left|g\left(t, z_{t}\right)\right|+1 .
$$

Then, for $z \in H$, we have

$$
|T z(t)|=\left|\int_{0}^{1} G(t, \theta) g\left(\theta, z_{\theta}\right) d \theta\right| \leq \frac{2 N}{(2-k) \Gamma(\beta)} .
$$

That is, $T(H)$ is uniformly bounded.

Let $z \in H$ and $t_{1}<t_{2}, t_{1}, t_{2} \in[-\tau, 1]$. If $0 \leq t_{1}<t_{2} \leq 1$, then

$$
\begin{aligned}
& \left|(T z)^{\prime}(t)\right| \\
& \quad=\left|-\int_{0}^{t} \frac{(t-\theta)^{\beta-2}}{\Gamma(\beta-1)} g\left(\theta, z_{\theta}\right) d \theta+\int_{0}^{1} \frac{2(1-\theta)^{\beta-1}(\beta-k+k \theta)}{(2-k) \Gamma(\beta+1)} g\left(\theta, z_{\theta}\right) d \theta\right| \\
& \quad \leq \int_{0}^{t} \frac{(t-\theta)^{\beta-2}}{\Gamma(\beta-1)}\left|g\left(\theta, z_{\theta}\right)\right| d \theta+\int_{0}^{1} \frac{2(1-\theta)^{\beta-1}(\beta-k+k \theta)}{(2-k) \Gamma(\beta+1)}\left|g\left(\theta, z_{\theta}\right)\right| d \theta \\
& \quad \leq N\left[\frac{t^{\beta-1}}{\Gamma(\beta)}+\frac{2(\beta+1-k)}{(2-k) \Gamma(\beta+2)}\right] \\
& \left.\quad \leq N \frac{\beta(\beta+1)(2-k)+2(\beta+1-k)}{(2-k) \Gamma(\beta+2)}\right]:=N_{0} .
\end{aligned}
$$

Hence

$$
\left|T z\left(t_{2}\right)-T z\left(t_{1}\right)\right| \leq \int_{t_{1}}^{t_{2}}\left|(T z)^{\prime}(\theta)\right| d \theta \leq N_{0}\left(t_{2}-t_{1}\right) .
$$


If $-\tau \leq t_{1}<t_{2} \leq 0$, then

$$
\left|T z\left(t_{2}\right)-T z\left(t_{1}\right)\right|=\left|\phi\left(t_{2}\right)-\phi\left(t_{1}\right)\right|
$$

If $-\tau \leq t_{1}<0<t_{2} \leq 1$, then

$$
\begin{aligned}
\left|T z\left(t_{2}\right)-T z\left(t_{1}\right)\right| & =\left|T z\left(t_{2}\right)-T z(0)\right|+\left|T z(0)-T z\left(t_{1}\right)\right| \\
& \leq \int_{0}^{1}\left|G\left(t_{2}, \theta\right)-G(0, \theta)\right|\left|g\left(\theta, z_{\theta}\right)\right| d \theta+\left|\phi(0)-\phi\left(t_{1}\right)\right| \\
& \leq \frac{2 N(\beta+1-k)}{(2-k) \Gamma(\beta+2)} t_{2}+\left|\phi(0)-\phi\left(t_{1}\right)\right| \\
& <\frac{2 N(\beta+1-k)}{(2-k) \Gamma(\beta+2)}\left|t_{2}-t_{1}\right|+\left|\phi(0)-\phi\left(t_{1}\right)\right| .
\end{aligned}
$$

Therefore, $T(H)$ is equicontinuous. On the basis of the Ascoli-Arzelà theorem we conclude that $T(H)$ is relatively compact. The conclusion has been proved.

Theorem 3.1 If $\left(C_{1}\right),\left(C_{2}\right),\left(C_{3}\right)$ and $\left(C_{5}\right)$ are satisfied, then Eq. (1) has at least two positive solutions $z_{1}$ and $z_{2}$ with

$$
0 \leq\left\|z_{1}\right\|_{[-\tau, 1]}<m<\left\|z_{2}\right\|_{[-\tau, 1]} .
$$

Proof Suppose that $\left(C_{5}\right)$ holds. Let $\Omega_{m}=\left\{z \in P:\|z\|_{[-\tau, 1]}<m\right\}$, for any $z \in P \cap \partial \Omega_{m}$, we have

$$
\begin{aligned}
(T z)(t) & = \begin{cases}\int_{0}^{1} G(t, \theta) g\left(\theta, z_{\theta}\right) d \theta, & 0 \leq t \leq 1, \\
\phi(t), & -\tau \leq t \leq 0,\end{cases} \\
& \leq \begin{cases}\frac{m}{B} M \int_{0}^{1} G(1, \theta) d \theta, & 0 \leq t \leq 1, \\
\|\phi\|_{[-\tau, 0]}, & -\tau \leq t \leq 0,\end{cases} \\
& \leq \begin{cases}m, & 0 \leq t \leq 1, \\
\|\phi\|_{[-\tau, 0]}, & -\tau \leq t \leq 0,\end{cases} \\
& \leq\|z\|_{[-\tau, 1]},
\end{aligned}
$$

which yields

$$
\|T z\|_{[-\tau, 1]} \leq\|z\|_{[-\tau, 1]}, \quad \text { for } z \in P \cap \partial \Omega_{m} .
$$

Suppose that $\left(C_{3}\right)$ holds. Since $g_{0}=\infty$, we may choose $\|\phi\|_{[-\tau, 0]}<m_{1}<m$, such that $g(t, z) \geq K\|z\|_{[-\tau, 0]}$, for $0 \leq\|z\|_{[-\tau, 0]} \leq m_{1}$, where $K>0$ satisfies $K A \geq 1$.

Let $\Omega_{m_{1}}=\left\{z \in P:\|z\|_{[-\tau, 1]}<m_{1}\right\}$, for any $z \in P \cap \partial \Omega_{m_{1}}$, we have

$$
(T z)\left(\frac{1}{2}\right) \geq \int_{\sigma}^{1-\sigma} G\left(\frac{1}{2}, \theta\right) g\left(\theta, z_{\theta}\right) d \theta \geq K \int_{\sigma}^{1-\sigma} G\left(\frac{1}{2}, \theta\right)\left\|z_{\theta}\right\|_{[-\tau, 0]} d \theta
$$




$$
\begin{aligned}
& \geq \frac{K \lambda}{2} \int_{\sigma}^{1-\sigma} G(1, \theta)\|z\|_{[0,1]} d \theta=\frac{K \lambda}{2} \int_{\sigma}^{1-\sigma} G(1, \theta)\|z\|_{[-\tau, 1]} d \theta \\
& \geq\|z\|_{[-\tau, 1]},
\end{aligned}
$$

which yields

$$
\|T z\|_{[-\tau, 1]} \geq\|z\|_{[-\tau, 1]}, \quad \text { for } z \in P \cap \partial \Omega_{m_{1}} .
$$

Next, since $g_{\infty}=\infty$, we may choose $m_{2}>m>\|\phi\|_{[-\tau, 0]}$, such that $g(t, z) \geq L\|z\|_{[-\tau, 0]}$, for $\|z\|_{[-\tau, 0]} \geq \lambda m_{2}$, where $L>0$ satisfies $L A \geq 1$.

Let $\Omega_{m_{2}}=\left\{z \in P:\|z\|_{[-\tau, 1]}<m_{2}\right\}$, for any $z \in P \cap \partial \Omega_{m_{2}}$, we have

$$
\begin{aligned}
(T z)\left(\frac{1}{2}\right) & \geq \int_{\sigma}^{1-\sigma} G\left(\frac{1}{2}, \theta\right) g\left(\theta, z_{\theta}\right) d \theta \geq L \int_{\sigma}^{1-\sigma} G\left(\frac{1}{2}, \theta\right)\left\|z_{\theta}\right\|_{[-\tau, 0]} d \theta \\
& \geq \frac{L \lambda}{2} \int_{\sigma}^{1-\sigma} G(1, \theta)\|z\|_{[0,1]} d \theta=\frac{L \lambda}{2} \int_{\sigma}^{1-\sigma} G(1, \theta)\|z\|_{[-\tau, 1]} d \theta \\
& \geq\|z\|_{[-\tau, 1]},
\end{aligned}
$$

which yields

$$
\|T z\|_{[-\tau, 1]} \geq\|z\|_{[-\tau, 1]}, \quad \text { for } z \in P \cap \partial \Omega_{m_{2}} .
$$

Therefore, the conclusion has been proved by (i) and (ii) of Lemma 2.4.

Theorem 3.2 If $\left(C_{1}\right),\left(C_{2}\right),\left(C_{4}\right)$ and $\left(C_{6}\right)$ are satisfied, then Eq. (1) has at least two positive solutions $z_{1}$ and $z_{2}$ with

$$
0 \leq\left\|z_{1}\right\|_{[-\tau, 1]}<n<\left\|z_{2}\right\|_{[-\tau, 1]} .
$$

Proof Suppose that $\left(C_{6}\right)$ holds. Letting $\Omega_{n}=\left\{z \in P:\|z\|_{[-\tau, 1]}<n\right\}$, for any $z \in P \cap \partial \Omega_{n}$, we have

$$
\begin{aligned}
(T z)\left(\frac{1}{2}\right) & \geq \int_{\sigma}^{1-\sigma} G\left(\frac{1}{2}, \theta\right) g\left(\theta, z_{\theta}\right) d \theta \geq \frac{1}{2} \int_{\sigma}^{1-\sigma} G(1, \theta) g\left(\theta, z_{\theta}\right) d \theta \\
& \geq \frac{n \lambda}{2 A} \int_{\sigma}^{1-\sigma} G(1, \theta) d \theta=n=\|z\|_{[-\tau, 1]}
\end{aligned}
$$

which yields

$$
\|T z\|_{[-\tau, 1]} \geq\|z\|_{[-\tau, 1]}, \quad \text { for } z \in P \cap \partial \Omega_{n} .
$$

Suppose that $\left(C_{4}\right)$ holds. Since $g_{0}=0$, we may choose $\|\phi\|_{[-\tau, 0]}<n_{1}<n$, such that $g(t, z) \leq$ $C\|z\|_{[-\tau, 0]}$, for $0 \leq\|z\|_{[-r, 0]} \leq n_{1}$, where $C>0$ satisfies $C B \leq 1$.

Let $\Omega_{n_{1}}=\left\{z \in P:\|z\|_{[-\tau, 1]}<n_{1}\right\}$, for any $z \in P \cap \partial \Omega_{m_{2}}$, we have

$$
(T z)(t)= \begin{cases}\int_{0}^{1} G(t, \theta) g\left(\theta, z_{\theta}\right) d \theta, & 0 \leq t \leq 1, \\ \phi(t), & -\tau \leq t \leq 0\end{cases}
$$




$$
\begin{aligned}
& \leq \begin{cases}C M \int_{0}^{1} G(1, \theta)\left\|z_{\theta}\right\|_{[-\tau, 0]} d \theta, & 0 \leq t \leq 1, \\
\|\phi\|_{[-r, 0]}, & -\tau \leq t \leq 0,\end{cases} \\
& \leq \begin{cases}C B\|z\|_{[-\tau, 1]}, & 0 \leq t \leq 1, \\
\|\phi\|_{[-r, 0]}, & -\tau \leq t \leq 0,\end{cases} \\
& \leq\|z\|_{[-\tau, 1]},
\end{aligned}
$$

which yields

$$
\|T z\|_{[-\tau, 1]} \leq\|z\|_{[-\tau, 1]}, \quad \text { for } z \in P \cap \partial \Omega_{n_{1}} .
$$

In addition, since $g_{\infty}=0$, there exists $R>n$, such that $g(t, z) \leq D\|z\|_{[-\tau, 0]}$, for $\|z\|_{[-\tau, 0]}>R$, where $D>0$ satisfies $(D+1) B \leq 1$.

Choose a constant $n_{2}>0$, such that $n_{2}>\max \left\{n,\|\phi\|_{[-\tau, 0]}, \max \left\{g\left(\theta, z_{\theta}\right) \mid 0 \leq\left\|z_{\theta}\right\|_{[-\tau, 0]} \leq\right.\right.$ $R\} B\}$. Let $\Omega_{n_{2}}=\left\{z \in P:\|z\|_{[-\tau, 1]}<n_{2}\right\}$, for any $z \in P \cap \partial \Omega_{n_{2}}$, we have

$$
\begin{aligned}
& (T z)(t) \\
& \leq\left\{\begin{array}{cc}
\int_{\left\|z_{\theta}\right\|_{[-\tau, 0]}>R} M G(1, \theta) g\left(\theta, z_{\theta}\right) d \theta \\
+\int_{0 \leq\left\|z_{\theta}\right\|_{[-\tau, 0]} \leq R} M G(1, \theta) g\left(\theta, z_{\theta}\right) d \theta, & 0 \leq t \leq 1, \\
\phi(t), & -\tau \leq t \leq 0,
\end{array}\right. \\
& \leq \begin{cases}\left\{D n_{2}+\max \left\{g\left(\theta, z_{\theta}\right) \mid 0 \leq\left\|z_{\theta}\right\|_{[-\tau, 0]} \leq R\right\}\right\} B, & 0 \leq t \leq 1, \\
\|\phi\|_{[-\tau, 0]}, & -\tau \leq t \leq 0,\end{cases} \\
& \leq \begin{cases}n_{2}, & 0 \leq t \leq 1, \\
\|\phi\|_{[-\tau, 0]}, & -\tau \leq t \leq 0,\end{cases} \\
& \leq n_{2}=\|z\|_{[-\tau, 1]},
\end{aligned}
$$

which yields

$$
\|T z\|_{[-\tau, 1]} \leq\|z\|_{[-\tau, 1]}, \quad \text { for } z \in P \cap \partial \Omega_{n_{2}} .
$$

Therefore, the conclusion has been proved by (i) and (ii) of Lemma 2.4.

From the ideas in the proofs of Theorem 3.1 and Theorem 3.2, we have Theorem 3.3 and Theorem 3.4.

Theorem 3.3 If $\left(C_{1}\right),\left(C_{2}\right)$ are satisfied and the conditions $g_{0}=\infty, g_{\infty}=0$ hold, then Eq. (1) has at least one positive solution.

Theorem 3.4 If $\left(C_{1}\right),\left(C_{2}\right)$ are satisfied and the conditions $g_{0}=0, g_{\infty}=\infty$ hold, then Eq. (1) has at least one positive solution. 


\section{Some examples}

Example 4.1 We consider the following equation:

$$
\left\{\begin{array}{l}
{ }^{c} D^{\frac{10}{3}} z(t)=-z^{\frac{1}{3}}\left(t-\frac{1}{2}\right), \quad t \in[0,1], \\
z(t)=t^{6}, \quad t \in\left[-\frac{1}{2}, 0\right], \\
z(0)=z^{\prime \prime}(0)=z^{\prime \prime \prime}(0)=0, \quad z(1)=\frac{1}{2} \int_{0}^{1} z(\theta) d \theta,
\end{array}\right.
$$

where $\alpha=\frac{10}{3}, k=\frac{1}{2}, \tau=\frac{1}{2}, g(t, z)=z^{\frac{1}{3}}\left(-\frac{1}{2}\right)$; since

$$
\frac{g(t, z)}{\|z\|_{\left[-\frac{1}{2}, 0\right]}}=\frac{z^{\frac{1}{3}}\left(-\frac{1}{2}\right)}{\|z\|_{\left[-\frac{1}{2}, 0\right]}} \leq \frac{\|z\|_{\left[-\frac{1}{2}, 0\right]}^{\frac{1}{3}}}{\|z\|_{\left[-\frac{1}{2}, 0\right]}}=\|z\|_{\left[-\frac{1}{2}, 0\right]}^{-\frac{2}{3}} \rightarrow 0, \quad \text { as }\|z\|_{\left[-\frac{1}{2}, 0\right]} \rightarrow+\infty
$$

we have $g_{\infty}=0$. In addition, there exists a constant $c>0$ with $z(t) \geq c\|z\|_{[-r, 0]}$,

$$
\frac{g(t, z)}{\|z\|_{\left[-\frac{1}{2}, 0\right]}}=\frac{z^{\frac{1}{3}}\left(-\frac{1}{2}\right)}{\|z\|_{\left[-\frac{1}{2}, 0\right]}} \geq c \frac{\|z\|_{\left[-\frac{1}{2}, 0\right]}^{\frac{1}{3}}}{\|z\|_{\left[-\frac{1}{2}, 0\right]}}=c\|z\|_{\left[-\frac{1}{2}, 0\right]}^{-\frac{2}{3}} \rightarrow+\infty, \quad \text { as }\|z\|_{\left[-\frac{1}{2}, 0\right]} \rightarrow 0 .
$$

Thus, $g_{0}=+\infty$, Eq. (6) has at least one positive solution by Theorem 3.3.

Example 4.2 We consider the following equation:

$$
\left\{\begin{array}{l}
{ }^{c} D^{\frac{7}{2}} z(t)=-\left(z^{\frac{1}{2}}\left(t-\frac{1}{3}\right)+z^{2}\left(t-\frac{1}{3}\right)\right), \quad t \in[0,1], \\
z(t)=t^{4}, \quad t \in\left[-\frac{1}{3}, 0\right], \\
z(0)=z^{\prime \prime}(0)=z^{\prime \prime \prime}(0)=0, \quad z(1)=\int_{0}^{1} z(\theta) d \theta,
\end{array}\right.
$$

where $\alpha=\frac{7}{2}, k=1, \tau=\frac{1}{3}, g(t, z)=z^{\frac{1}{2}}\left(-\frac{1}{3}\right)+z^{2}\left(-\frac{1}{3}\right)$, and there exists a constant $c>0$ with $z(t) \geq c\|z\|_{[-r, 0]}$; since

$$
\begin{aligned}
& \frac{g(t, z)}{\|z\|_{\left[-\frac{1}{3}, 0\right]}}=\frac{z^{\frac{1}{2}}\left(-\frac{1}{3}\right)+z^{2}\left(-\frac{1}{3}\right)}{\|z\|_{\left[-\frac{1}{3}, 0\right]}} \geq c \frac{\|z\|_{\left[-\frac{1}{3}, 0\right]}^{\frac{1}{2}}+\|z\|_{\left[-\frac{1}{3}, 0\right]}^{2}}{\|z\|_{\left[-\frac{1}{3}, 0\right]}} \rightarrow+\infty, \quad \text { as }\|z\|_{\left[-\frac{1}{3}, 0\right]} \rightarrow+\infty, \\
& \frac{g(t, z)}{\|z\|_{\left[-\frac{1}{3}, 0\right]}}=\frac{z^{\frac{1}{2}}\left(-\frac{1}{3}\right)+z^{2}\left(-\frac{1}{3}\right)}{\|z\|_{\left[-\frac{1}{3}, 0\right]}} \geq c \frac{\|z\|_{\left[-\frac{1}{3}, 0\right]}^{\frac{1}{2}}+\|z\|_{\left[-\frac{1}{3}, 0\right]}^{2}}{\|z\|_{\left[-\frac{1}{3}, 0\right]}} \rightarrow+\infty, \quad \text { as }\|z\|_{\left[-\frac{1}{3}, 0\right]} \rightarrow 0,
\end{aligned}
$$

we have $g_{0}=+\infty, g_{\infty}=+\infty$. Thus the condition $\left(C_{3}\right)$ holds. Furthermore, $M=\frac{2 \alpha}{k(\alpha-2)}=$ $\frac{14}{3}, \int_{0}^{1} G(1, s) d s=\int_{0}^{1} \frac{(1-s)^{\alpha-1}(k[2(s-1)+\alpha])}{(2-k) \Gamma(\alpha+1)} d s=\frac{5}{9 \Gamma\left(\frac{9}{2}\right)}, B=M \int_{0}^{1} G(1, s) d s=\frac{224}{567 \sqrt{\pi}}$. Taking $m=2$, then when $0 \leq\|z\|_{\left[-\frac{1}{3}, 0\right]} \leq 2$, we have $g(t, z) \leq 6 \leq \frac{m}{B}=\frac{567 \sqrt{\pi}}{112}$, which implies the condition $\left(C_{5}\right)$ holds. Hence by Theorem 3.1, Eq. (7) has at least two positive solutions $z_{1}$ and $z_{2}$ with

$$
0<\|z\|_{\left[-\frac{1}{3}, 1\right]}<2<\|z\|_{\left[-\frac{1}{3}, 1\right]} .
$$

\section{Conclusion}

In this paper, on the basis of the Guo-Krasnoselskii theorem, the sufficient conditions ensure that the existence and multiplicity of positive solutions are obtained. This research 
method can be extended to many fractional boundary value problems. It is worth noting that the equation involves time delay and an integral boundary value condition, to be compared to much previous work, which has never been considered. In addition, our work is inspiring for future research as regards triple positive solutions of fractional boundary value problems with delay.

\section{Acknowledgements}

The authors acknowledge the support by the National Natural Science Foundation of China (11601003; 11371027; 11471015)

\section{Funding}

This work is supported by the National Natural Science Foundation of China (no.11601003, no.11371027 and no.11471015)

Availability of data and materials

Not applicable.

\section{Competing interests}

The authors declare that they have no competing interests.

Authors' contributions

All authors contributed equally to this paper. All authors read and approved the final manuscript.

\section{Publisher's Note}

Springer Nature remains neutral with regard to jurisdictional claims in published maps and institutional affiliations.

Received: 28 February 2020 Accepted: 14 May 2020 Published online: 02 June 2020

\section{References}

1. Podlubny, I.: Fractional Differential Equations. Academic Press, San Diego (1999)

2. Lakshmikantham, V., Guo, D.J.: Nonlinear Problems in Abstract Cone. Academic Press, Orlando (1988)

3. Kilbas, A.A., Srivastava, H.M., Trujillo, J.J.: Theory and Applications of Fractional Differential Equations. Elsevier, Amsterdam (2006)

4. Oldham, K.B., Spanier, J.: The Fractional Calculus. Academic Press, New York (1974)

5. Lakshmikantham, V., Leela, S., Vasundhara, D.J.: Theory of Fractional Dynamic Systems. Cambridge Academic Publishers, Cambridge (2009)

6. Diethelm, V.: The Analysis of Fractional Differential Equations. Springer, New York (2010)

7. Iqbal, Z., Ahmed, N., Baleanu, D., Rafiq, M., Iqbal, M.S., Rehman, M.A.: Structure preserving computational technique for fractional order Schnakenberg model. Comput. Appl. Math. 39(2), 61 (2020)

8. Hajipour, M., Jajarmi, A., Malek, A., Baleanu, D.: Positivity-preserving sixth-order implicit finite difference weighted essentially non-oscillatory scheme for the nonlinear heat equation. Appl. Math. Comput. 325, 146-158 (2018)

9. Ahmed, N., Rafiq, M., Baleanu, D., Rehman, M.A.: Spatio-temporal numerical modeling of auto-catalytic Brusselator model. Rom. J. Phys. 64(7-8), 110 (2019)

10. Zhao, Y., Sun, S., Han, Z., Zhang, M.: Positive solutions for boundary value problems of nonlinear fractional differential equations. Appl. Math. Comput. 217(16), 6950-6958 (2011)

11. Feng, M.Q., Zhang, X.M., Ge, W.G.: New existence results for higher-order nonlinear fractional differential equation with integral boundary conditions. Bound. Value Probl. 2011, Article ID 720702 (2011)

12. Qiao, Y., Zhou, Z.F.: Existence and uniqueness of positive solutions for a fractional differential equation with integral boundary conditions. Adv. Differ. Equ. 2016, 106 (2016)

13. Qiao, Y., Zhou, Z.F.: Existence of solutions for a class of fractional differential equations with integral and anti-periodic boundary conditions. Bound. Value Probl. 2017, 11 (2017)

14. Ji, D.H.: Positive solutions of singular fractional boundary value problem with p-Laplacian. Bull. Malays. Math. Sci. Soc. 41, 249-263 (2018)

15. Wang, Y.Q., Liu, L.S.: Positive solutions for a class of fractional infinite-point boundary value problems. Bound. Value Probl. 2018, $118(2018)$

16. Tian, Y.S., Bai, Z.B., Sun, S.J.: Positive solutions for a boundary value problem of fractional differential equation with p-Laplacian operator. Adv. Differ. Equ. 2019, 349 (2019)

17. Sun, Q., Ji, H.W., Cui, Y.J.: Positive solutions for boundary value problems of fractional differential equation with integral boundary conditions. J. Funct. Spaces Appl. 2018, Article ID 6461930 (2018)

18. Zhang, D.L., Liu, Y.S.: Multiple positive solutions for nonlinear fractional boundary value problems. Sci. World J. 2013, Article ID 473828 (2013)

19. Zhang, X.Q., Zhong, Q.Y.: Multiple positive solutions for nonlocal boundary value problems of singular fractional differential equations. Bound. Value Probl. 2016, 65 (2016)

20. Zhao, K.H., Wang, K.: Existence of solutions for the delayed nonlinear fractional functional differential equations with three-point integral boundary value conditions. Adv. Differ. Equ. 2016, 284 (2016)

21. Avery, R.I., Peterson, A.C.: Three positive fixed points of nonlinear operators on ordered Banach spaces. Comput. Math. Appl. 42(3-5), 313-322 (2001) 
22. Guo, L.M., Zhang, X.Q.: Existence of positive solutions for the singular fractional differential equations. J. Appl. Math. Comput. 44, 215-228 (2014)

23. Yang, L., Shen, C.F., Xie, D.P.: Multiple positive solutions for nonlinear boundary value problem of fractional order differential equation with the Riemann-Liouville derivative. Adv. Differ. Equ. 2014, 284 (2014)

24. Li, Y.H.: Multiple positive solutions for nonlinear mixed fractional differential equation with $p$-Laplacian operator. Adv. Differ. Equ. 2019, 112 (2019)

25. Li, Y.N., Sun, S.R., Yang, D.W., Han, Z.L.: Three-point boundary value problems of fractional functional differential equations with delay. Bound. Value Probl. 2013, 38 (2013)

26. Niazi, A.U.K., Jiang, W., Rehman, M.U., Pang, D.H.: Boundary value problem for nonlinear fractional differential equations with delay. Adv. Differ. Equ. 2017, 24 (2017)

27. Jawaz, M., Ahmed, N., Baleanu, D., Rehman, M.A.: Positivity preserving technique for the solution of HIV/AIDS reaction diffusion model with time delay. Front. Phys. 7, 229 (2020)

28. Cabada, A., Wang, G.T.: Positive solutions of nonlinear fractional differential equations with integral boundary value conditions. J. Math. Anal. Appl. 389(1), 403-411 (2012)

29. Han, Z.L., Li, Y.A., Sui, M.Z.: Existence results for boundary value problems of fractional functional differential equations with delay. J. Appl. Math. Comput. 51(1-2), 367-381 (2016)

\section{Submit your manuscript to a SpringerOpen ${ }^{\circ}$ journal and benefit from:}

- Convenient online submission

- Rigorous peer review

- Open access: articles freely available online

- High visibility within the field

- Retaining the copyright to your article

Submit your next manuscript at $\boldsymbol{\nabla}$ springeropen.com 